\title{
MEMORE - Um Ambiente para Apoio ao Acompanha- mento da Utilização de Computadores na Educação
}

\author{
MEMORE - An Environment for Monitoring the Use of Computers in Education \\ Ronaldo Goldschmidt \\ Universidade Federal Rural do Rio de Janeiro \\ ronaldo.rgold@gmail.com \\ Claudio Passos \\ Colégio Pedro II \\ cpassos.cp2@gmail.com

\section{Claudia Ferlin} \\ Fundação de Apoio à Escola Técnica do Rio de Janeiro \\ claudia.ferlin@gmail.com \\ Isabel Fernandes \\ Universidade Federal Rural do Rio de Janeiro \\ isabelfs.ufrrj@gmail.com \\ Mônica Norris \\ Secretaria de Educação de Piraí \\ mncnorris@gmail.com \\ Maria Claudia Cavalcanti \\ Instituto Militar de Engenharia \\ maryoko@gmail.com
}

Jorge Soares

Universidade do Estado do Rio de Janeiro

jasoares@gmail.com

Resumo A utilização do computador como ferramenta educacional é um tema de grande relevância na sociedade moderna. Neste contexto, o governo federal brasileiro lançou o programa 'Um Computador por Aluno' (PROUCA), que vem promovendo a implantação de laptops para uso educacional nas escolas. A iniciativa PROUCA carece de instrumentos que permitam às instâncias gestoras conhecer, acompanhar e avaliar ações pedagógicas vinculadas à utilização dos laptops em sala de aula e em atividades extraclasse. Assim sendo, este artigo tem como objetivo apresentar o MEMORE, um ambiente computacional concebido de forma a prover informações gerenciais a respeito de como os laptops PROUCA têm sido usados pelos beneficiários do programa. Tais informações podem ser utilizadas por docentes e gestores para apoiar a avaliação e o aprimoramento de políticas educacionais e práticas pedagógicas nas escolas.

Palavras-Chave: Tecnologias Educacionais; Projeto Um Computador por Aluno; Sistemas de Apoio à Decisão.

\begin{abstract}
The use of computers as teaching and learning tools plays a particularly important role in modern society. Within this scenario, Brazil launched its own version of the 'One Laptop per Child' (OLPC) program, and this initiative, termed PROUCA, has already distributed hundreds of lowcost laptops for educational purposes in many Brazilian schools. However, in spite of its importance, PROUCA program does not have tools that help managers monitor, understand and evaluate which curricular and extracurricular activities have been developed with the laptops. Therefore, this article aims to present MEMORE, a computational environment that provides management information about the use of laptops in PROUCA's context. Teachers and administrators can take such information into consideration in order to evaluate and improve educational policies and pedagogical practices in schools.
\end{abstract}

Keywords: Educational Technologies; One Laptop per Child Programs; Decision Support Systems 


\section{Introdução}

Os constantes avanços das Tecnologias da Informação e Comunicação (TIC) têm proporcionado diferentes recursos computacionais com aplicação prática e robusta em diversos segmentos da Sociedade [3,18]. Em particular, a utilização do computador como ferramenta educacional é um tema de grande relevância e que tem sido objeto de pesquisa multidisciplinar envolvendo principalmente as áreas da Educação e da Computação [36].

Inspirados na iniciativa "One Laptop per Child" (OLPC) do Laboratório de Mídias do Instituto de Tecnologia de Massachusetts (MIT), diversos países vêm implantando programas voltados à distribuição de computadores de baixo custo nas escolas [22]. A maioria deles é motivada pela possibilidade de que o uso das TIC pelos alunos conduza a melhorias no aprendizado e na produtividade acadêmica [13].

Neste contexto, o governo federal brasileiro lançou, inicialmente, o Projeto Um Computador por Aluno (UCA) e, em seguida, o Programa Um Computador por Aluno (PROUCA). Ambos vêm promovendo a implantação de laptops de baixo custo para uso educacional nas escolas como estímulo à inclusão digital de discentes, docentes e gestores [19]. Visando a simplificação do texto, as duas iniciativas serão referenciadas indistintamente ao longo deste artigo pela sigla PROUCA, e correspondem à versão brasileira do Programa OLPC.

No entanto, somente a distribuição de computadores pelas escolas, assim como a implantação de laboratórios e o treinamento de docentes, não é suficiente para assegurar o uso pedagógico desses recursos de forma plena e satisfatória [21]. Diante desse cenário, os gestores do PROUCA carecem de informações consolidadas sobre as práticas pedagógicas cotidianas envolvendo a utilização dos laptops distribuídos [4]. Apesar de todos os estudos realizados no país desde o lançamento do PROUCA $[5,9,10,19,28,29,31,33]$, o Brasil desconhece, por exemplo, quantos computadores estão sendo efetivamente utilizado, o tempo médio de uso dos laptops pelos alunos de cada escola, a distribuição desse tempo por disciplina, onde os computadores têm sido usados e o desempenho escolar dos alunos beneficiados pelo programa, dentre várias outras informações básicas importantes para a gestão e o aprimoramento de qualquer iniciativa OLPC.

Assim sendo, o presente artigo tem como objetivo descrever um ambiente computacional denominado Mecanismo de Monitoramento Remoto (MEMORE) que foi concebido para apoiar docentes e gestores no aprimoramento de políticas educacionais e práticas pedagógicas no contexto PROUCA. Elaborado com base em princípios de Inteligência Artificial [27] e Mineração de Dados
[11], o MEMORE coleta, integra, consolida e disponibiliza continuamente diversos dados da realidade escolar como, por exemplo, índices de desempenho (IDEB, notas e frequências das turmas) e informações acerca do uso dos laptops pelos beneficiários do PROUCA (tempos de utilização por disciplina, por software, por local de acesso, por tipo de atividade desenvolvida, dentre outras). Além das informações técnicas sobre o MEMORE, este trabalho apresenta ainda uma série de resultados obtidos com o uso do ambiente proposto em duas escolas públicas brasileiras.

Este texto encontra-se organizado em mais quatro seções. A seção 2 apresenta uma revisão bibliográfica sobre iniciativas de investigação e avaliação do impacto de programas voltados à distribuição de computares nas escolas em diversos países. A metodologia de desenvolvimento e a modelagem de algumas das principais funcionalidades disponibilizadas pelo MEMORE são relatadas na seção 3. A seção 4 descreve os experimentos realizados no ano de 2012 e os principais resultados obtidos com o projeto até o momento. Considerações finais sobre o trabalho assim como perspectivas de ações futuras estão indicadas na seção 5 .

\section{Trabalhos Relacionados}

Segundo Trucano [4], o impacto do uso das TICs para a melhoria do ensino nas escolas é de difícil aferição e permanece como um tema aberto ao debate e à pesquisa.

São raras as iniciativas de investigação dos resultados obtidos a partir de programas voltados à distribuição de laptops de baixo custo a crianças e jovens nos diversos países do mundo [13]. Além disso, a maioria dos estudos existentes foi conduzida em países desenvolvidos [13] e apresenta diferentes abordagens metodológicas com diferentes enfoques, critérios e níveis de rigor científico [24].

Uma revisão detalhada e recente da literatura sobre a avaliação de programas OLPC pelo mundo pode ser obtida em [22]. Os autores reportam que tais programas, incluindo o PROUCA brasileiro, concentram suas avaliações em estudos de caso específicos cujas coletas de dados para análise, quando existem, são pontuais e isoladas, inviabilizando um acompanhamento de longo prazo. Em muitos casos, existe pouca documentação formal disponível sobre as avaliações realizadas. Com exceção de planilhas eletrônicas e enquetes via web, em nenhum dos programas retratados por Nugroho e Longsdale [22] foi possível identificar a existência de ferramentas informatizadas que proporcionassem apoio à captação contínua, ao armazenamento centralizado e à análise de longo prazo de dados oriundos da utilização dos computadores fornecidos aos estudantes. 
Ainda segundo Nugroho e Longsdale [22], na avaliação dos programas OLPC, as coletas de dados para estudo são, em geral, realizadas por meio de questionários de cunho qualitativo, e muitas vezes subjetivo, onde o público alvo deve reportar, no momento da pesquisa, informações tais como: a faixa de frequência com que determinados aplicativos foram utilizados, a opinião sobre o quanto a aluno aprendeu sobre algum tema, o quanto as aulas se tornaram mais atrativas, dentre inúmeras outras [17].

Diante deste cenário, o MEMORE se apresenta como um ambiente computacional de origem brasileira para apoio à automatização do processo de coleta contínua, armazenamento centralizado, análise e disponibilização sistematizada de informações pedagógicas e operacionais para gestores educacionais e grupos de pesquisa. Ao captar os dados diretamente da utilização dos laptops, o MEMORE pode reduzir a margem de erro decorrente da subjetividade de questionários de avaliação qualitativa dos OLPCs comum em vários países. Além disso, o ambiente proposto não impõe uma abordagem metodológica de pesquisa, oferecendo flexibilidade aos pesquisadores, gestores educacionais e patrocinadores na escolha entre diferentes opções de técnicas e algoritmos para estudo dos dados segundo diversos interesses e níveis de detalhe ao longo do tempo.

O sistema MONITOR é uma solução comercial para a administração de ambientes de redes de computadores [20]. Inclui funcionalidades para coleta e análise de informações sobre o hardware (tais como: estatísticas de computadores ativos, inativos e defeituosos de uma escola) e sobre a comunicação de dados (como, por exemplo, estatísticas sobre o volume de dados transmitidos, em um determinado período, por uma escola ou por um computador específico). Oferece ainda facilidades para execução de rotinas de instalação e atualização remotas de software. Tal solução vem sendo adotada em diversas escolas do PROUCA, porém, diferentemente do MEMORE, não permite o monitoramento de aspectos pedagógicos, fundamentais para avaliação e ajuste da evolução do desempenho escolar. Portanto, MONITOR e MEMORE se apresentam como soluções complementares para apoiar políticas educacionais envolvendo um computador por aluno.

Especificamente no contexto do PROUCA, escolas e pesquisadores envolvidos nas experiências iniciais do programa sinalizaram para a importância e a necessidade de monitoramento e avaliação dos projetos educacionais envolvendo os laptops distribuídos. Foi, então, lançado um guia de implementação, monitoramento e avaliação de projetos UCA. Esse documento, denominado Relatório de Sistematização III, embora oriente para práticas de verificação sistemática e periódica de questões como ambiente escolar, infraestrutura, armazena- mento e segurança [19], não aponta para soluções computacionais que auxiliem na captura e armazenamento dessas informações. De forma análoga, Fonseca [10] também contribui com questões importantes a serem consideradas na avaliação da infraestrutura do PROUCA. Diante de ambos os trabalhos, o MEMORE se mostra como uma alternativa de apoio para automatização do processo de coleta, disponibilização e análise dessas e de outras informações de natureza pedagógica para docentes, gestores educacionais e grupos de pesquisa.

Ainda no Relatório de Sistematização III do PROUCA seguem recomendações para acompanhamento da evolução do IDEB das escolas [5]. Novamente com relação a este item, o MEMORE se revela como uma iniciativa complementar que facilita a realização do acompanhamento recomendado, por meio de consultas que permitem uma confrontação entre os valores de IDEB apurados ao longo dos anos e as metas originalmente estabelecidas para cada escola.

\section{Solução Proposta}

\subsection{Metodologia de desenvolvimento}

O ambiente MEMORE é fruto de uma experiência de construção coletiva envolvendo universidades, instâncias de governo e escolas públicas. Surgiu a partir do interesse do governo federal brasileiro em conhecer com detalhes as práticas pedagógicas que vêm sendo desenvolvidas com a utilização dos laptops nas diversas escolas do PROUCA [4].

A proposta do projeto foi inicialmente concebida em parceria por um grupo de pesquisa composto por pesquisadores de cinco universidades brasileiras.

A concepção de um sistema de informação requer uma etapa inicial de levantamento de requisitos [23]. Tomando como base a demanda geral estabelecida pelo CNPq [4], houve a necessidade de busca por escolas que pudessem tanto apoiar o processo de modelagem de requisitos quanto atuar como piloto nos testes e na validação do MEMORE.

Por ser um dos municípios brasileiros com maior experiência no cenário do PROUCA, Piraí no Estado do Rio de Janeiro aderiu à proposta do projeto. A Secretaria de Educação de Piraí indicou duas escolas para atuar no trabalho. A escolha das escolas procurou atender às duas modalidades do OLPC brasileiro, proporcionando maior representatividade do contexto de estudo. Desta forma, uma das escolas selecionadas está ligada ao projeto UCA (CIEP 477 Professora Rosa da Conceição Guedes) e a outra é beneficiada pela iniciativa PROUCA (E.M. Rosa Carelli da Costa). A equipe gestora da Secretaria de Educação de Piraí selecionou quatro turmas piloto do ensino fundamental para o projeto, sendo quatro do primeiro 
segmento e quatro do segundo.

Quatro tipos de reunião de divulgação do projeto foram realizados em cada escola: (a) junto à equipe gestora; (b) junto a todo corpo docente; (c) junto aos discentes de cada turma piloto; (d) junto aos responsáveis pelos discentes das turmas piloto.

Em todas as reuniões foram apresentados os objetivos do projeto e a metodologia proposta. Materiais específicos e com linguagem apropriada a cada público alvo foram criados e adotados. Nas reuniões junto aos responsáveis, estes foram solicitados a assinar um termo formal autorizando a participação dos discentes no projeto.

Em relação aos aspectos técnicos, o Práxis [23] foi o processo de software adotado na criação do MEMORE. A linguagem de modelagem utilizada foi a UML [2].

Diversas reuniões de levantamento e análise de requisitos foram realizadas junto à Secretaria Municipal de Educação e às escolas piloto. As turmas piloto foram envolvidas. Mais de cento e vinte reuniões de trabalho sobre o projeto foram realizadas. As técnicas de Brainstorming e de Participatory Design [23] foram adotadas em vários momentos durante a concepção do sistema. $\mathrm{O}$ projeto das interfaces dos agentes de coleta de dados com os beneficiários do PROUCA foi construído com o apoio de técnicas de modelagem de Interface HumanoComputador [25, 26,34]. Sessões de observação de aulas em que os alunos utilizaram os laptops também foram necessárias e realizadas [9].

O processo de Verificação e Validação (V\&V) [8] do MEMORE ocorreu durante todo o ciclo de desenvolvimento do ambiente. Técnicas de inspeção dos artefatos de software [25] foram constantemente empregadas nas fases de levantamento de requisitos, modelagem e projeto do sistema. Vários casos de teste foram elaborados e utilizados nas fases de programação e validação do MEMORE. Os registros de aprovação dos requisitos e modelos do sistema foram documentados em atas de reuniões específicas para a execução do processo V\&V. Também foram realizadas reuniões para homologação do software, além da oferta de cursos de treinamento dos usuários na utilização do ambiente.

Todo o desenvolvimento do ambiente proposto foi baseado nos princípios da orientação a objetos [32,39].

A modelagem de dados do MEMORE foi inspirada na abordagem de identificação de atributos baseada em pontos cardeais. Esta abordagem é muito usual na construção de sistemas multidimensionais utilizados em processos de apoio à decisão [15]. Consiste em identificar dimensões (atributos) a partir de perguntas-chave tais como “quem?”, “quando?”, “onde?”, “o que?”. No contexto do MEMORE, essas questões foram adaptadas para levantar informações sobre, por exemplo, sessões de utilização dos laptops: "quem usou?", “quando usou?", “onde usou?" e "o que usou?".

Cabe ressaltar ainda que, em função da natureza multidisciplinar do projeto MEMORE, o desenvolvimento e a implantação do ambiente proporcionaram a integração de equipes de áreas distintas. De um lado, colaboradores com experiência em computação abrangendo pesquisadores, analistas de sistemas, programadores e alunos de graduação. Do outro, professores, gestores e técnicos com um sólido histórico de atuação na área da educação básica. Foram muitas as oportunidades de troca de conhecimentos e experiências entre os envolvidos [9].

Em vários momentos, o processo de desenvolvimento do MEMORE levou a interferências na dinâmica das turmas envolvidas. Portanto, o trabalho certamente extrapolou o Práxis e, no decorrer do processo, percebeuse o exercício da pesquisa-ação [3738]. Fernandes et al. [9] apresenta um maior detalhamento sobre a experiência de construção do MEMORE.

\subsection{Modelagem desenvolvida}

A figura 1 apresenta uma visão geral simplificada do modelo funcional do MEMORE, destacando algumas das principais funcionalidades oferecidas pelo ambiente. Abaixo encontram-se comentadas cada uma delas.

O MEMORE foi projetado para atender às seguintes classes de usuários: (a) Beneficiários do programa que são estudantes ou professores contemplados com laptops fornecidos pelo PROUCA; (b) Diretores das escolas e demais gestores educacionais envolvidos no programa; (c) Analistas de dados que são profissionais capacitados a aplicar técnicas e algoritmos de mineração sobre os dados coletados; (d) Visitantes são pessoas não vinculadas oficialmente ao PROUCA, mas que têm interesse em conhecer práticas e resultados gerados pelo programa.

Inspirado em [10], o caso de uso "Relatar Situação Operacional da Escola" permite ao diretor da escola descrever, por meio de um questionário específico, as condições operacionais de sua instituição diante do PROUCA. A tabela 1 apresenta alguns exemplos de questões que devem ser respondidas pelo gestor em seu relato. 


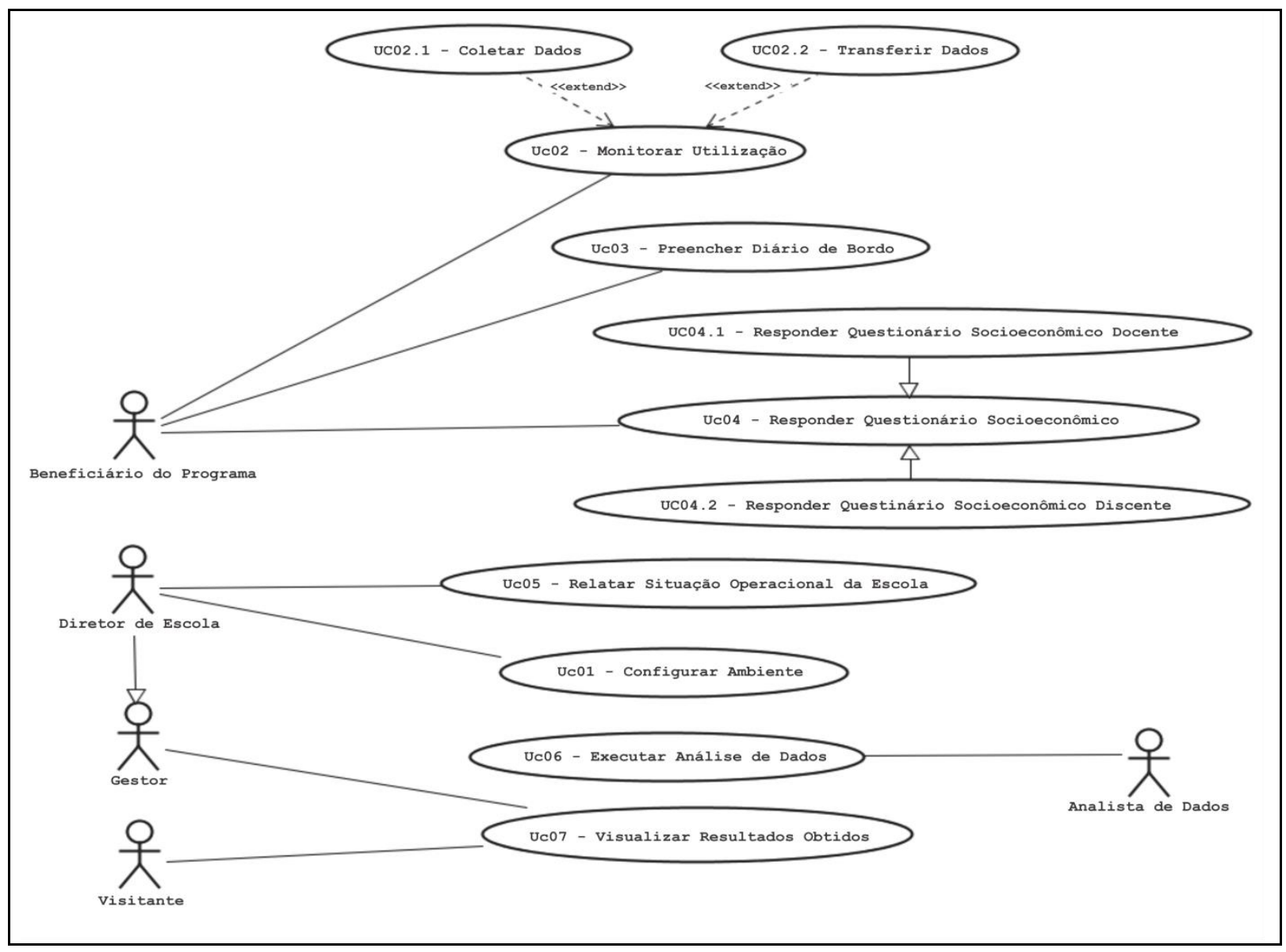

Figura 1 : Modelo Funcional do Ambiente Memore - Visão Geral Simplificada.

Uma das mais importantes funcionalidades do ambiente MEMORE, o caso de uso "Monitorar Utilização" consiste de dois agentes puramente reativos [27], sendo um associado ao caso de uso "Coletar Dados" (agente de coleta de dados) e o outro vinculado ao caso de uso "Transferir Dados" (agente de transferência de dados).

O agente de coleta de dados tem como objetivo perceber o momento em que cada software é iniciado a fim de registrar a data e a hora desse evento. Adicionalmente, esse mesmo agente também é responsável por registrar a data e a hora em que cada software é encerrado. Para desempenhar sua função, esse agente precisa conhecer a relação dos softwares selecionados pela escola que devem ser monitorados e ser capaz de acompanhar os eventos do sistema operacional a fim de detectar as situações de inicialização e encerramento dos softwares selecionados.

Quando o usuário utiliza o navegador web, o agente de coleta de dados identifica e registra todos os endereços eletrônicos visitados durante a navegação.

No encerramento de cada software monitorado, o agente de coleta de dados interage com o beneficiário a fim de enriquecer as informações sobre a utilização do recurso. Nesse momento, sempre que a utilização tenha sido para fins acadêmicos, o agente indaga, por exemplo, sobre as disciplinas envolvidas, o tipo de atividade realizada, a forma de trabalho (individual ou em grupo) e o local de utilização do laptop. As respostas do usuário são armazenadas em um arquivo de log, junto com os horários de inicialização e encerramento de cada software acessado durante a sessão de utilização. $\mathrm{O}$ arquivo de log é identificado pelo número de série do laptop onde foi gerado.

O conteúdo da interação entre o agente de coleta de dados e o usuário varia de acordo com o tipo do usuário. Por exemplo, o conjunto de atividade executadas por docentes é diferente do conjunto de atividades executadas por discentes. Como o PROUCA atende a todos os níveis da Educação Básica, entre os discentes, existem também alunos em fase de alfabetização. A interação entre o agente de coleta de dados e esse conjunto de alunos precisa ser diferenciada. Para atender a esse público específico, o agente de coleta de dados foi projetado para utilizar imagens e sons como recursos de apoio complementa- 
res às perguntas formuladas com texto. Fernandes et al. [9] apresenta a experiência piloto de aplicação do agente de coleta de dados junto a alunos em fase de alfabetização.

Foram criadas, portanto, três versões do agente de coleta de dados. Cada versão voltada a um público específico: alunos alfabetizados, alunos em alfabetização e docentes. Assim, antes da entrega de cada laptop ao beneficiário responsável, é realizada a instalação da versão do agente de coleta de dados compatível com o tipo de receptor.

O agente de transferência de dados, por sua vez, monitora o conteúdo de uma pasta previamente especificada e executa, periodicamente, o processo de envio dos dados coletados para um banco de dados central único (denominado central de análise), sempre que determinadas condições (volume mínimo de informações coletadas e disponibilidade de internet) são atendidas.

No início de cada sessão de utilização, o agente de transferência verifica se há disponibilidade de acesso à internet pelo laptop. Em caso positivo, naquele momento, o agente transporta para a central de análise os dados disponíveis no arquivo de log gerado pelo agente de cole- ta. O processo de transferência ocorre de forma imperceptível pelo usuário do laptop.

Conforme o próprio nome sugere, o caso de uso "Responder Questionário Socioeconômico" permite que docentes e discentes informem dados tais como faixa de renda familiar, local de residência, faixa etária, dentre outros. Este caso de uso encontra-se especializado em dois outros, adequados ao respectivo público alvo: "Responder Questionário Socioeconômico Docente" e "Responder Questionário Socioeconômico Discente".

O caso de uso "Preencher Diário de Bordo" permite que docentes, discentes e gestores relatem experiências cotidianas no contexto do PROUCA. Para tanto, diante da metáfora de um diário, os usuários do MEMORE podem registrar de forma livre e textual fatos e/ou opiniões sobre o programa e suas vivências.

Tanto o preenchimento dos questionários quanto a atualização dos diários de bordo contribuem para enriquecer o banco de dados do MEMORE na central de análise. Essas informações são integradas aos históricos de dados obtidos pelo módulo de coleta de dados. Consequentemente podem ser levadas em consideração nos processos de análise e mineração de dados.

\begin{tabular}{l|l|l}
\hline Assunto & Enunciado da Questão & Respostas Possíveis \\
\hline Segurança & $\begin{array}{l}\text { A escola dispõe de grades nas janelas e portas ade- } \\
\text { quadas à segurança dos laptops? }\end{array}$ & Sim / Dispõe parcialmente / Não \\
\hline Infraestrutura & $\begin{array}{l}\text { A velocidade de acesso à internet atende à demanda } \\
\text { da escola: }\end{array}$ & $\begin{array}{l}\text { Plenamente / Parcialmente / Precari- } \\
\text { amente / Não atende }\end{array}$ \\
\hline Capacitação & $\begin{array}{l}\text { Professores, gestores e técnicos da escola tiveram } \\
\text { treinamento inicial na utilização dos dispositivos } \\
\text { didáticos recebidos? }\end{array}$ & Sim / Estão em treinamento / Não \\
\hline Suporte Técnico & $\begin{array}{l}\text { A escola dispõe de apoio técnico operacional de } \\
\text { informática às atividades pedagógicas relacionadas } \\
\text { ao PROUCA (aulas, reuniões, trabalhos, etc...)? }\end{array}$ & $\begin{array}{l}\text { Sim, plenamente satisfatório / Sim, } \\
\text { parcialmente satisfatório / Não }\end{array}$
\end{tabular}

Tabela 1: Exemplos de questões do relato da situação operacional da escola

O caso de uso "Executar Análise de Dados" oferece algoritmos de pré-processamento e de mineração de dados ao analista, que tem autonomia para selecionar e aplicar os que forem necessários. Um dos algoritmos disponíveis no ambiente MEMORE é o Apriori [1], um clássico exemplo para execução da tarefa de Mineração de Regras de Associação. Esta tarefa procura identificar conjuntos de informações que ocorrem de forma simultânea e frequente em um banco de dados. Formalmente, uma regra de associação é uma implicação da forma: (Ri) $\mathrm{X}==>\mathrm{Y}$, onde: (a) $\mathrm{X}$ e $\mathrm{Y}$ são conjuntos de itens não vazios tais que $\mathrm{X} \cap \mathrm{Y}=\varnothing$; (b) o suporte da regra, calculado por $\sup \left(\mathrm{R}_{\mathrm{i}}\right)=\mid \mathrm{X}$ e $\mathrm{Y}|/| \mathrm{D} \mid$ (a proporção do número de vezes que os itens de $\mathrm{X}$ e de $\mathrm{Y}$ ocorrem simultaneamente em relação ao total de casos disponíveis no conjunto de dados), deve ser maior ou igual a um suporte mínimo estabelecido pelo usuário (SupMin); (c) a confiança da regra, expressa por $\operatorname{conf}\left(\mathrm{R}_{\mathrm{i}}\right)=\mid \mathrm{X}$ e $\mathrm{Y}|/| \mathrm{X} \mid$ (a proporção do número de vezes que os itens de $\mathrm{X}$ e de $\mathrm{Y}$ ocorrem juntos em relação ao total de casos em que os itens do antecedente de $\mathrm{R}_{\mathrm{i}}$ ocorrem no conjunto de dados), deve ser maior ou igual a uma confiança mínima estabelecida pelo usuário (ConfMin).

O caso de uso "Configurar Ambiente" é fundamental para a operacionalização do MEMORE. Por meio dele, todas as informações básicas necessárias ao funcionamento do sistema devem ser alimentadas. 
Inclui, por exemplo, o cadastramento de usuários e seus direitos de acesso, a inserção de escolas e de concessões dos laptops para os beneficiários. Também é responsável por importar os valores de IDEB de cada escola e por receber os dados referentes ao desempenho acadêmico obtido pelos alunos em cada disciplina ao longo do ano letivo, além da taxa de evasão escolar.

Por fim, o caso de uso "Visualizar Resultados Obtidos" permite que gestores educacionais, diretores de escola, docentes e visitantes tenham acesso aos dados coletados e analisados com o apoio do MEMORE. A apresentação dos resultados é feita por meio de relatórios. Esses relatórios encontram-se organizados nos seguintes grupos: (a) Resultados das análises de dados - neste grupo estão os relatórios gerados a partir das principais e mais relevantes conclusões obtidas com o apoio do caso de uso "Executar Análise de Dados". (b) Consultas gerenciais - este grupo contém dados estatísticos consolidados sobre os principais assuntos disponíveis no MEMORE. São exemplos desses assuntos: utilização dos laptops; situações operacionais das escolas no contexto do PROUCA; desempenho de turmas; evolução do IDEB (índice de desenvolvimento da educação básica [14]); perfis socioeconômicos de docentes e de discentes; entre outros.

\subsection{Protótipo}

O governo brasileiro adotou o Classmate como modelo de laptop no PROUCA. Com uma configuração simplificada de 512 MB de RAM e 4 GB de HD, os laptops possuem como sistema operacional uma distribuição Linux adaptada do sistema Megoo.

O desenvolvimento dos agentes de coleta e de transferência de dados foi direcionado para que esses pudessem operar na configuração descrita. As linguagens de programação utilizadas foram C [16,30], Python [35] e Bash [6].

Considerando o alto custo computacional típico de aplicações de Mineração de Dados, o caso de uso "Executar Análise de Dados" deve operar em ambiente local. Sua implementação foi desenvolvida em linguagem C com banco de dados PostgreSQL [12]. Os requisitos mínimos recomendados para a plataforma de execução dessa implementação são $2 \mathrm{~GB}$ de RAM e $20 \mathrm{MB}$ de disco.

Os demais casos de uso foram desenvolvidos em PHP [7] para operar na web e facilitar o acesso aos dados pelos gestores educacionais. Foram configurados dois servidores, sendo um voltado ao armazenamento da aplicação e o outro para a hospedagem do banco de dados. Ambos se encontram sob a segurança do PRODERJ, órgão público responsável pela área de TIC no estado do
Rio de Janeiro no Brasil. O sistema gerenciador de banco de dados escolhido também foi o PostgreSQL.

As figuras 2(a) e 2(b) apresentam visões parciais das interfaces de interação do agente de coleta de dados com alunos alfabetizados e com alunos em alfabetização, respectivamente.

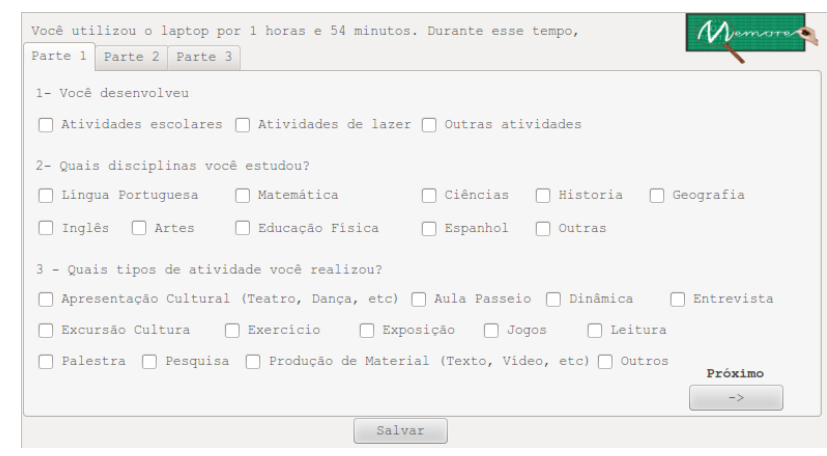

Figura 2a: Interface entre agente de coleta de dados e o aluno alfabetizado.

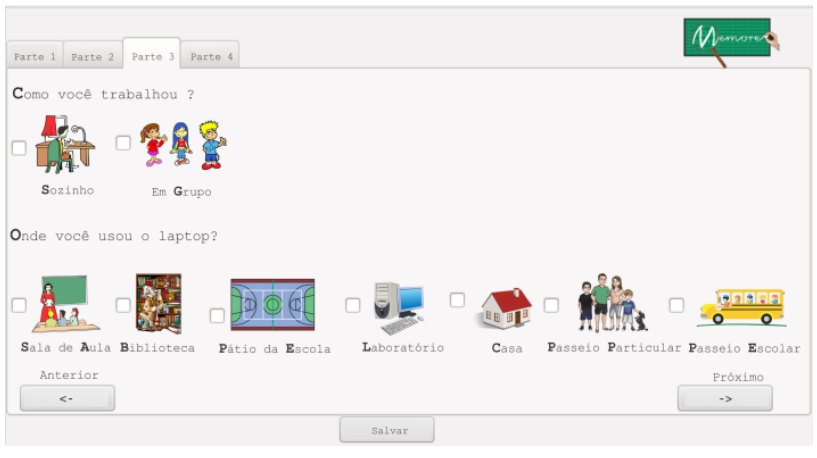

Figura 2b: Interface entre agente de coleta de dados e o aluno em alfabetização.

Por meio do questionário da figura 3(a), diretores podem relatar aspectos operacionais do PROUCA em suas escolas. Informações sobre o perfil socioeconômico de docentes são coletadas pelo questionário ilustrado na figura $3(\mathrm{~b})$.

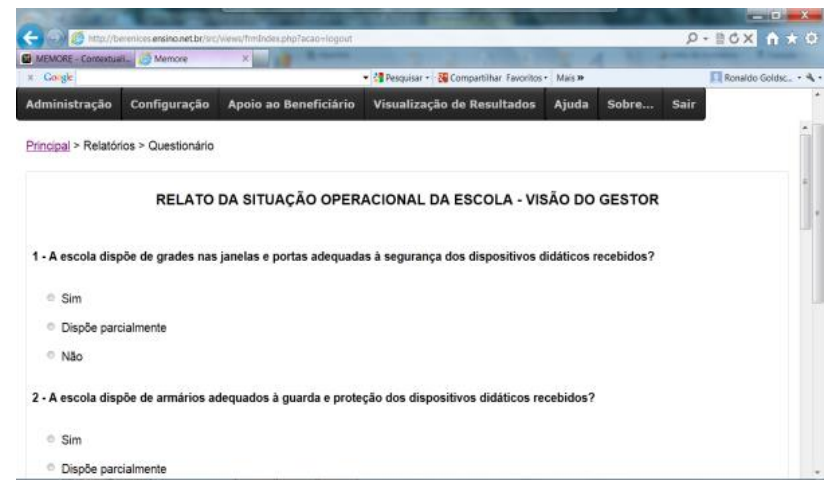

Figura 3a: Interface do questionário sobre a situação operacional da escola. 


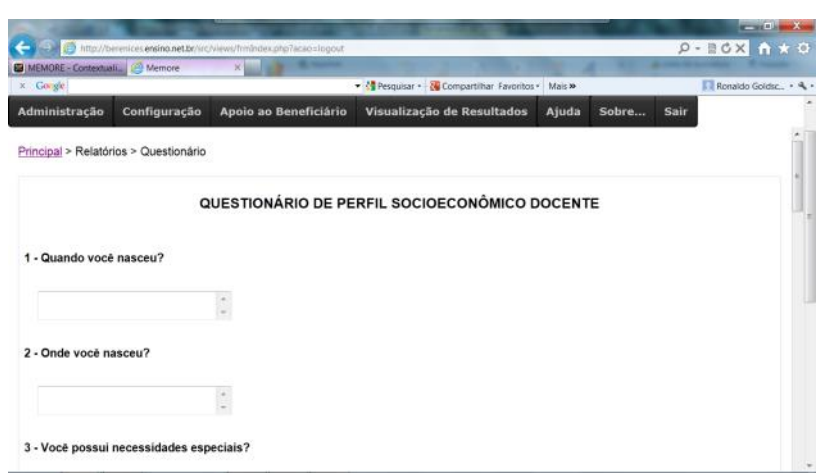

Figura 3b: Interface do questionário sobre o perfil socioeconômico docente.

Um exemplo de relatório gerencial produzido pelo ambiente encontra-se ilustrado na figura 4 .

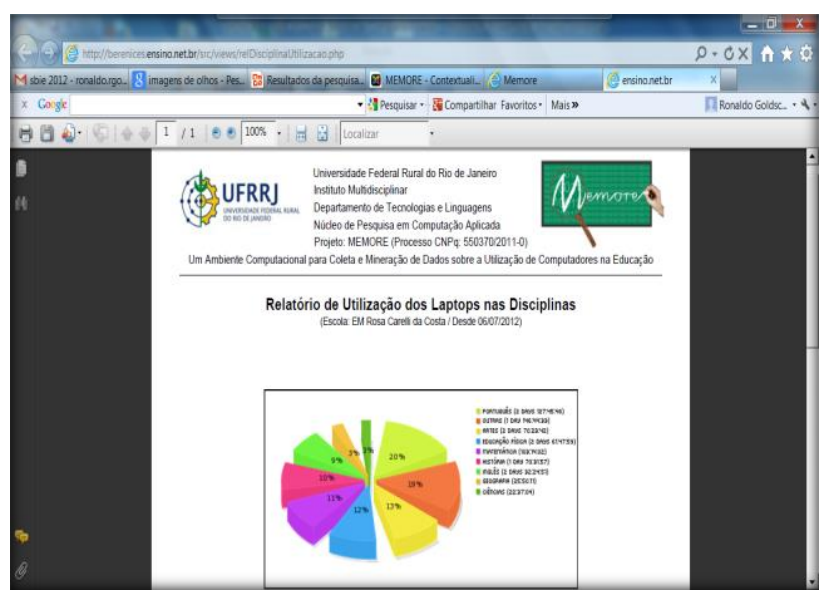

Figura 4: Relatório gerencial sobre a distribuição do tempo de uso dos laptops por disciplina.

\section{Resultados e Discussões}

Esta seção tem como objetivos apresentar e comentar exemplos de resultados produzidos pelo MEMORE que ilustram o potencial do ambiente em suprir carências de informação dos gestores educacionais brasileiros sobre o desenvolvimento do PROUCA.

Para os experimentos descritos nesta seção, os agentes de coleta e de transferência de dados foram implantados em sessenta e sete laptops distribuídos pelas quatro turmas piloto indicadas. Todos os alunos das turmas piloto participaram do experimento. A coleta ocorreu no período de 01 de agosto a 31 de outubro de 2012 .

Do início do período de coleta até o final de setembro de 2012, os computadores eram mantidos nas escolas, conforme regra geral para todas as escolas do município. Após essa data, todos os alunos do município foram autorizados pela Secretaria Municipal de Educação a levar diariamente os laptops para suas casas. Ao final do ano letivo, os computadores foram devolvidos às escolas para manutenção.

Cabe ressaltar que, durante o período da coleta, surgiram algumas demandas por manutenção dos laptops das turmas piloto. No entanto, como as escolas dispunham de uma equipe técnica de suporte, o tempo de manutenção dos equipamentos nunca foi superior a dois dias úteis.

Os dados coletados foram transferidos, integrados e consolidados em um banco de dados único (central de análise). Apuramos um total de 1.549 horas, 6 minutos e 9 segundos de uso em 1.413 sessões de utilização. Com base nesses dados, estimamos uma média de quase duas horas de utilização por semana, por laptop. (Memória de cálculo aproximado: $1.549 / 1.413=1$ hora por sessão; 1.549 horas $/ 67=23$ horas por laptop; $23 / 3$ meses $=7$ horas por mês $=1,8$ horas por semana).

A tabela 2 apresenta a distribuição dos laptops, do número de sessões de uso e do tempo total de utilização pelas quatro turmas piloto. Alunos mais velhos utilizaram os laptops por mais tempo. A utilização pelos mais novos, sobretudo os da turma 201 (com faixa etária em torno dos seis anos e em fase de alfabetização) ficou mais restrita aos momentos assistidos pela professora em sala de aula.

Cabe destacar que apenas 3\% do tempo médio de utilização dos laptops durante o período observado foi dedicado ao desenvolvimento de atividades em grupo. Este número sobe para $18 \%$ quando restringimos o universo de observação apenas às sessões de utilização realizadas em sala de aula. Esta variação indica que o volume de demanda por atividades em grupo foi maior em tarefas de sala de aula do que em tarefas extraclasse.

Os softwares com maior tempo médio de utilização no período analisado foram: Chromium (15 min e 42 seg), Tux Paint (14 min e $41 \mathrm{seg})$, LibreOffice Writer (12 min e $40 \mathrm{seg}$ ) e GCompris (08 min e $09 \mathrm{seg}$ ), nesta ordem. Em função da alta demanda pela internet, o aplicativo mais acessado foi, como esperado, o navegador web Chromium. O uso recorrente de software para desenho (Tux Paint) e escrita (LibreOffice Writer) também não surpreende. São aplicativos associados a atividades acadêmicas frequentes na Educação Fundamental. A popularidade do GCompris também justifica a razoável utilização do software. Trata-se de um pacote de software educativo contendo jogos de alta atratividade junto ao público infanto-juvenil.

Em 4,4\% das sessões de utilização dos laptops, os alunos afirmaram que tiveram muitas dúvidas na manipulação dos recursos computacionais. Em 16,6\% dos casos, declararam poucas dúvidas do uso do computador. Ne- 
nhuma dúvida foi relatada nas demais sessões de utilização (79\% dos casos). Esses dados evidenciam um bom proporcionada pelos laptops. nível de assimilação dos alunos em relação à tecnologia

\begin{tabular}{c|c|c|c|c}
\hline Turma & \# Laptops/Alunos & Idade Média & \# Sessões & Tempo Total (Horas) \\
\hline 201 & 13 & Seis anos & 120 & $136: 03$ \\
\hline 402 & 18 & Nove anos & 201 & $317: 31$ \\
\hline 701 & 19 & Doze anos & 634 & $702: 01$ \\
\hline 801 & 17 & Quatorze anos & 431 & $393: 13$
\end{tabular}

Tabela 2: Distribuição dos laptops e tempos de utilização pelas turmas piloto.

As figuras 5(a) e 5(b) mostram, respectivamente, a distribuição do tempo médio de utilização dos laptops por disciplina e por local de utilização no período observado. A alta incidência de utilizações em passeios escolares reflete as várias atividades realizadas pelas turmas piloto junto à comunidade local, próxima à escola.

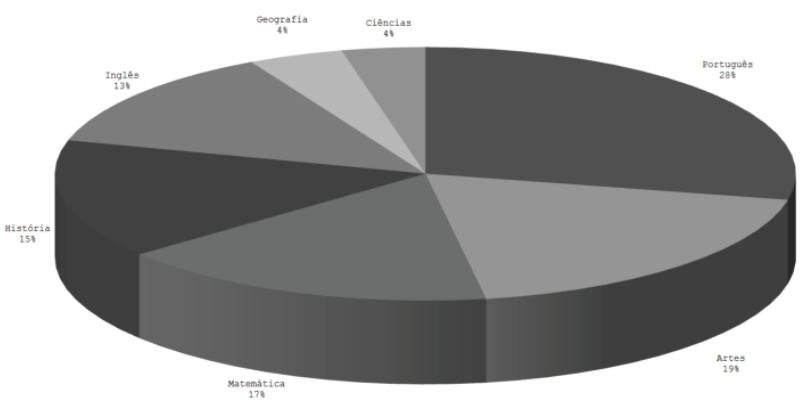

Figura 5a: Tempo médio de uso dos laptops - Distribuição por disciplina.

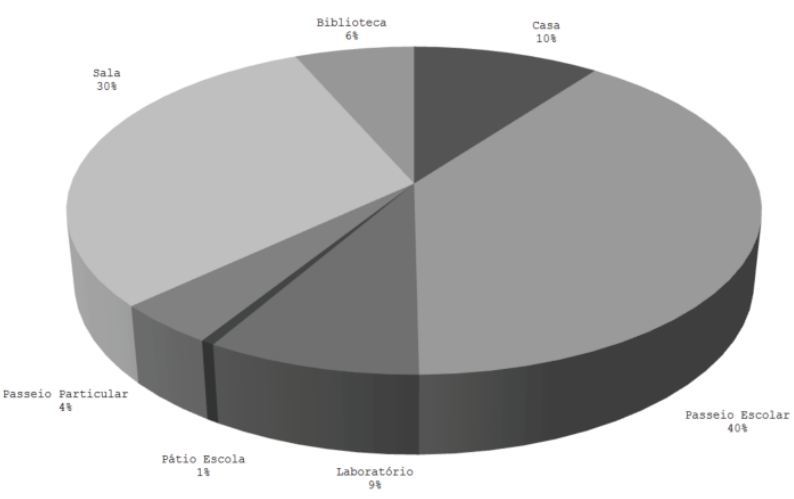

Figura 5b: Tempo médio de uso dos laptops - Distribuição por local de uso.

A figura 6 apresenta um retrato da evolução do desempenho médio dos alunos das turmas piloto. As turmas foram avaliadas bimestralmente. Embora o intervalo de avaliação tenha extrapolado o período de coleta de dados do projeto, o gráfico representa um instrumento para acompanhamento longitudinal das turmas. As médias apresentadas na figura foram obtidas pelos alunos na disciplina de Matemática.

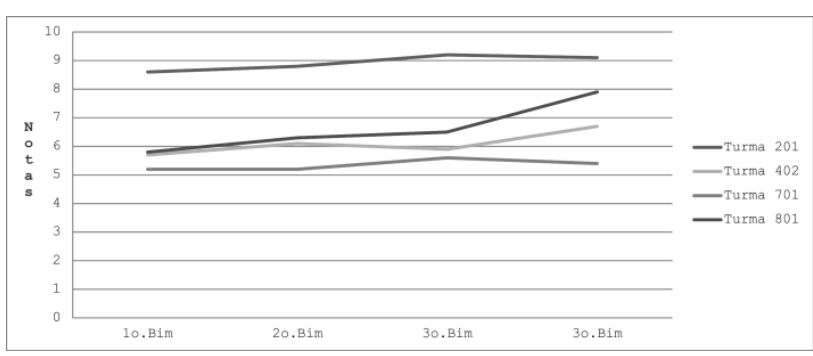

Figura 6: Evolução do Desempenho Acadêmico Médio das Turmas Piloto em Matemática.

A figura 7 contém outro exemplo de visualização de dados proporcionada pelo MEMORE. Os dados se referem ao tempo de utilização dos laptops por duas das turmas piloto. Essa forma de apresentação dos dados permite, além do acompanhamento longitudinal do tempo de utilização, uma comparação entre o comportamento de uso das turmas selecionadas pelo usuário no momento da formulação da consulta no sistema.

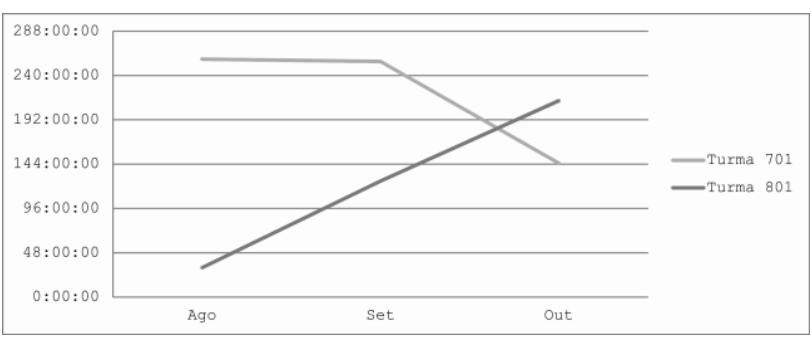

Figura 7: Evolução do Tempo de Utilização dos Laptops por Turma Piloto.

Os gestores das duas escolas piloto preencheram, cada um, o relato das condições operacionais de sua escola. Esse preenchimento ocorreu no final do primeiro semestre de 2012. A tabela 3 exibe um sumário com os principais aspectos operacionais identificados nas escolas pesquisadas.

Em outubro de 2012, vinte e três professores das escolas piloto (incluindo os docentes das turmas piloto) responderam ao questionário de perfil socioeconômico disponibilizado pelo MEMORE. A figura 8 mostra alguns 
resultados apurados neste contexto.

Principais Aspectos Operacionais das Escolas Piloto

A segurança dos laptops foi considerada parcialmente satisfatória em ambas as escolas.

Os armários para recarga dos laptops são adequados e atendem à demanda.

A internet foi considerada estável e com velocidade satisfatória em ambas as escolas.

Uma das escolas carece de coordenador pedagógico interno responsável pelo PROUCA.

Uma das escolas carece de apoio técnico de informática às atividades pedagógicas do PROUCA.

Nas duas escolas, professores, gestores e técnicos recebem treinamento contínuo no uso dos laptops.

Tabela 3: Principais aspectos operacionais levantados nas escolas piloto $(07 / 2012)$.

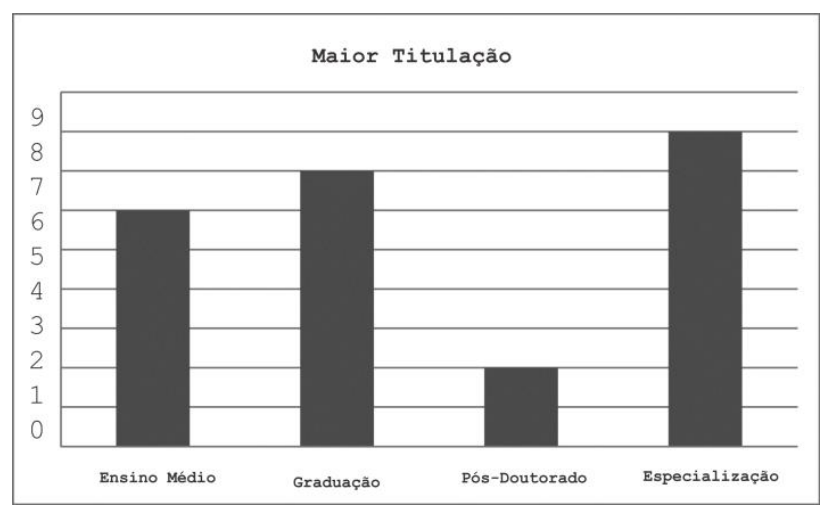

Figura 8a: Exemplo de característica de perfil socioeconômico docente - Maior Titulação.

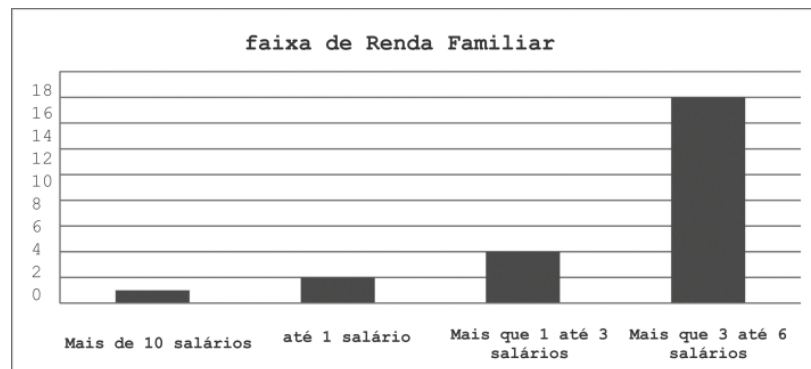

Figura 8b: Exemplo de característica de perfil socioeconômico docente - Renda Familiar.

A tabela 4 apresenta alguns exemplos de regras de associação produzidas a partir da aplicação do algoritmo Apriori sobre o conjunto de dados reunidos na central de análise. Foram utilizados os valores $3 \%$ e $70 \%$ como suporte e confiança mínimos, respectivamente. A regra $\mathrm{R}_{1}$, por exemplo, indica que em todos os casos que os alunos trabalharam sozinhos e utilizaram o Chromium como uma de suas ferramentas, eles conseguiram com- pletar todas as suas atividades. A regra $\mathrm{R}_{2}$ revela que todas as atividades de lazer durante o período observado foram desenvolvidas individualmente pelos alunos. A regra $R_{5}$ mostra que em $88 \%$ de todas as vezes que os alunos utilizaram os laptops para desenvolver atividades de Matemática, tais atividades foram concluídas. Segundo a regra $\mathrm{R}_{9}$, em $96 \%$ das sessões de utilização realizadas em casa, os alunos conseguiram concluir suas atividades. As regras $R_{10}$ e $R_{11}$ sugerem um surpreendente indicativo de equilíbrio entre os tipos de atividades desenvolvidas em casa: $46 \%$ foram atividades de laser e $43 \%$ atividades escolares. Elas contrariam a expectativa dos gestores educacionais do município piloto de que, em casa, o uso dos laptops seria fortemente direcionado para atividades de lazer.

\begin{tabular}{|c|c|c|}
\hline Regra & Sup & Conf \\
\hline $\begin{array}{l}\left(\mathrm{R}_{1}\right) \text { Atuou individualmente e utilizou o } \\
\text { Google Chromium }==>\text { Concluiu todas as } \\
\text { atividades desenvolvidas }\end{array}$ & 7,3 & 100 \\
\hline $\begin{array}{l}\left(\mathrm{R}_{2}\right) \text { Desenvolveu atividade de lazer ==> } \\
\text { Desenvolveu individualmente }\end{array}$ & 6,9 & 100 \\
\hline $\begin{array}{l}\left(\mathrm{R}_{3}\right) \text { Desenvolveu atividades individual- } \\
\text { mente e utilizou o Google Chromium ==> } \\
\text { Concluiu todas as atividades e não teve } \\
\text { dificuldade na utilização dos recursos }\end{array}$ & 6,9 & 95 \\
\hline $\begin{array}{l}\left(\mathrm{R}_{4}\right) \text { Desenvolveu atividade de lazer }==> \\
\text { Não apresentou dificuldade de utilização }\end{array}$ & 6,5 & 94 \\
\hline $\begin{array}{l}\left(\mathrm{R}_{5}\right) \text { Desenvolveu atividade de Matemáti- } \\
\mathrm{ca}==>\text { Conseguiu concluir atividade }\end{array}$ & 3,1 & 88 \\
\hline $\begin{array}{l}\left(\mathrm{R}_{6}\right) \text { Desenvolveu atividade de Ciências } \\
==>\text { Conseguiu concluir atividade }\end{array}$ & 2,7 & 86 \\
\hline $\begin{array}{l}\left(\mathrm{R}_{7}\right) \text { Desenvolveu atividade de Língua } \\
\text { Portuguesa ==> Conseguiu concluir ativi- } \\
\text { dade }\end{array}$ & 3,4 & 78 \\
\hline $\begin{array}{l}\left(\mathrm{R}_{8}\right) \text { Atuou em grupo, não utilizou para } \\
\text { lazer e desenvolveu atividades escolares } \\
==>\text { Concluiu todas as atividades desen- } \\
\text { volvidas }\end{array}$ & 3,4 & 82 \\
\hline $\begin{array}{l}\left(\mathrm{R}_{9}\right) \text { Atuou em casa }==>\text { Concluiu todas as } \\
\text { atividades desenvolvidas }\end{array}$ & 3,3 & 96 \\
\hline $\begin{array}{l}\left(\mathrm{R}_{10}\right) \text { Atuou em casa }==>\text { Desenvolveu } \\
\text { atividades escolares }\end{array}$ & 3,5 & 43 \\
\hline $\begin{array}{l}\left(\mathrm{R}_{11}\right) \text { Atuou em casa }==>\text { Desenvolveu } \\
\text { atividade de lazer }\end{array}$ & 3,6 & 46 \\
\hline $\begin{array}{l}\left(\mathrm{R}_{12}\right) \text { Atuou em grupo }==>\text { Desenvolveu } \\
\text { atividades escolares }\end{array}$ & 3,3 & 100 \\
\hline
\end{tabular}

Tabela 4: Regras de Associação geradas pelo Apriori (SupMin=3\%; ConfMin $=70 \%$ )

O MEMORE permite ainda que o gestor educacional possa comparar a evolução dos valores de IDEB observados com metas projetadas, destacando o ano de implantação do PROUCA em cada escola. A tabela 5 mostra um 
quadro comparativo do desempenho das duas escolas 2009.

piloto em relação às metas projetadas para o IDEB de

\begin{tabular}{c|c|c|c|c|c|c|c}
\hline Escola & $\begin{array}{c}\text { Início } \\
\text { PROUCA }\end{array}$ & $\begin{array}{c}\text { Meta IDEB } \\
5^{\circ} \text {. ano }\end{array}$ & $\begin{array}{c}\text { IDEB Real } \\
5^{\circ} \text {. ano }\end{array}$ & $\begin{array}{c}\text { Dif. } \\
5^{\circ} \text {. Ano }\end{array}$ & $\begin{array}{c}\text { Meta IDEB } \\
9^{\circ} \text {. Ano }\end{array}$ & $\begin{array}{c}\text { IDEB Real } \\
9^{\circ} \text {. Ano }\end{array}$ & $\begin{array}{c}\text { Dif. } \\
9^{\circ} \text {. Ano }\end{array}$ \\
\hline Guedes & 2007 & 3,1 & 4,5 & 31,1 & 4,1 & 4,5 & 8,9 \\
\hline Carelli & 2011 & 4,3 & 3,4 & $-26,5$ & 3,6 & 4,0 & 10,0
\end{tabular}

Tabela 5: Quadro comparativo entre IDEB observado e metas das escolas piloto.

\section{Considerações Finais}

Em sintonia com vários países em desenvolvimento, o governo federal brasileiro lançou o programa Um Computador por Aluno (PROUCA) que tem como objetivo de promover a implantação de laptops para uso educacional nas escolas como estímulo à inclusão digital de discentes, docentes e gestores [19]. No entanto, a iniciativa PROUCA carece de instrumentos que permitam aos gestores educacionais conhecer, acompanhar e avaliar ações pedagógicas vinculadas ao uso dos laptops nas salas de aula e fora delas [4].

Diante desse cenário, o presente artigo teve como objetivo apresentar o MEMORE, um ambiente computacional que é resultado de uma integração de esforços do Governo Federal, universidades e escolas públicas em busca da construção de instrumentos que auxiliem os gestores educacionais no acompanhamento e na avaliação do PROUCA. Para tanto, o artigo descreveu o processo de desenvolvimento do MEMORE e algumas das principais funcionalidades do ambiente. Experimentos iniciais foram relatados, assim como seus respectivos resultados, ilustrando assim o potencial do MEMORE para atuar como sistema de informações gerenciais no apoio à avaliação e ao aprimoramento de políticas educacionais e práticas pedagógicas no contexto PROUCA.

Embora concebido inicialmente como um instrumento tecnológico, o MEMORE abre oportunidades para o desenvolvimento de diversas pesquisas científicas no contexto da computação aplicada à educação. Assim, além da evolução natural do MEMORE como sistema de informação gerencial, são possibilidades de trabalhos futuros: uma busca por métricas que permitam avaliar o impacto do PROUCA considerando os princípios norteadores do programa tais como mobilidade, conectividade e imersão; uma análise comparativa entre os projetos de aprendizagem planejados pelos professores e os efeitos práticos decorrentes da execução desses projetos; a construção de uma rede social voltada ao compartilhamento da base de dados do MEMORE junto a escolas e à comunidade científica em geral; uma investigação em busca de relações entre o uso dos laptops e o desempenho acadêmico dos alunos; a aplicação de técnicas de mineração de textos sobre os depoimentos textuais livres coletados por meio do preenchimento dos questionários do MEMORE pelos beneficiários do programa; um estudo detalhado das questões éticas envolvidas com a captura e uso das informações do PROUCA, dentre outras.

\section{Agradecimentos}

Este trabalho recebe fomento do Conselho Nacional de Pesquisa (CNPq) sob o processo número: 550370/2011-0. O Centro de Tecnologia da Informação e Comunicação do Estado do Rio de Janeiro (PRODERJ) é responsável pela hospedagem gratuita dos servidores do projeto.

\section{Referências}

[1] Agrawal, R., Imielinski, T. and Swami, A. (1993) Mining Association Rules Between Sets of Items in Large Databases. In: Proceedings of the 1993 ACM SIGMOD Conference Management of Data. Washington DC.

[2] Bezerra, E. (2007) Princípios de Análise e Projeto de Sistemas com UML. $2^{\text {a }}$ ed., Rio de Janeiro: Campus.

[3] Castells, M. (2009) A Era da Informação: Economia, Sociedade e Cultura. Coleção A Sociedade em Rede. 12a . Reimpressão. São Paulo: Paz e Terra.

[4] CNPq/CAPES/SEED-MEC (2010) Edital no. $76 / 2010$. PROUCA Fase 2. Available at $<$ http://memoria.cnpq.br/editais/ct/2010/docs/07 6.pdf>, access on 5 July 2012.

[5] Coelho, F. and Jardim, M. H. (2010) Relatório de Sistematização III - Guia de Implementação, Monitoramento e Avaliação. Programa UCA. Available at <http://www.uca.gov.br/institucional/downloads/ experimentos/DFguiaImplementacao.pdf>, ac- 
cess on 5 July 2012.

[6] Costa, D. G. (2010) Administração de Redes com Scripts: BASH SCRIPT, PYTHON E VBSCRIPT. Rio de Janeiro: Brasport.

[7] Dall'Oglio, P. (2007) PHP: Programando com Orientação a Objetos. São Paulo: Novatec.

[8] Delamaro, M. E., Maldonado and J. C., Jino, M. (2007) Introdução ao Teste de Software. Rio de Janeiro: Elsevier.

[9] Fernandes, I., Goldschmidt, R., Norris, M. and Silva, R. (2012) Relato de uma Experiência de Integração Universidade- Governo-Escola na Construção Coletiva de um Sistema de Monitoramento do Programa Um Computador por Aluno. In: Anais do XXIII SBIE- XVIII WIE. Rio de Janeiro: SBC.

[10] Fonseca, A. L. B. (2011) Projeto UCA - Um Computador por Aluno: Analisando as Condições da Implantação em uma Escola da Rede Pública do Distrito Federal. Trabalho Final de Curso. Licenciatura em Pedagogia, Faculdade de Educação/UnB.

[11] Goldschmidt, R. and Passos, E. (2005) Data Mining: Um Guia Prático. Rio de Janeiro: Campus.

[12] Gonzaga, J. L. (2007) Dominando o PostgreSQL. Rio de Janeiro: Ciência Moderna.

[13] Hansen, N. et al. (2012) Laptop Usage Affects Abstract Reasoning of Children in the Developing World. Computers\& Education 59: 9891000 .

[14] INEP (2012) Censo Escolar da Educação Básica Brasileira. Available at <http://portal.inep.gov.br/basica-censo>, access on 10 May 2013.

[15] Inmon, W. (2005) Building the Data Warehouse. Indianapolis, Indiana: Wiley Publishing.

[16] Kernighan, B.W. (1984) The Unix Programming Environment. New Jersey: Prentice-Hall.

[17] Kozma, R. et al. (2004) Closing the Digital Divide: Evaluation of the World Links Program. International Journal of Educational Development 24: 361-381.
[18] Laurindo, F. J. B. (2008) Tecnologia da Informação: Planejamento e Gestão de Estratégias. São Paulo: Atlas.

[19] Meneses, S. C. P. (2011) UCA - Um Computador por Aluno: Era da Inclusão Digital, Anais do XXII SBIE- XVII WIE. Aracaju: SBC. Available at <http://www.br-ie.org/sbie-wie2011/SBIETrilha7/92966_1.pdf>, access on 5 July 2012.

[20] Measys, (2012) "Metasys Monitor". http://www.metasys.com.br/index.php?option=c om_content $\&$ view $=$ article $\&$ id=293\&Itemid $=165$ \&lang $=\mathrm{pt}$

[21] Motta, R. A. S. M., Santos, T. A. C., Goldschmidt, R. R., Campos, M. F. (2009) Escola Mandala em Ação. Rio de Janeiro: Imprinta.

[22] Nugroho, D., Longsdale, M. (2010) Evaluation of OLPC Programs Globally: A Literature Review (Version 4). Australian Council for Education Research. Available at <http://wiki.laptop.org/images/a/a5/olpc_Lit_Re view_v4_Aug2010.pdf $>$, access on 12 July 2012.

[23] Paula Filho, W. P. (2005) Engenharia de Software. Rio de Janeiro: LTC.

[24] Penuel, W. R. (2006) Implementation and Effects of One-to-One Computing Initiatives. Journal of Research on Technology in Education 38: 329-348.

[25] Pressman, R. S. (2006) Engenharia de Software. $6^{a}$. ed. São Paulo: McGraw-Hill.

[26] Rocha, H. V. and Baranauskas, M. C. (2003) Design e Avaliação de Interfaces HumanoComputador. Campinas: NIED/UNICAMPI.

[27] Russell, S. J. and Norvig, P. (2004) Inteligência Artificial. $2^{\mathrm{a}}$ ed. Rio de Janeiro: Campus.

[28] Sampaio, F. and Elia, M. (orgs) (2012) Projeto um Computador Por Aluno: Pesquisas e Perspectivas. Rio de Janeiro: NCE/UFRJ.

[29] Sampaio, F. and Miranda, L. C. (orgs) (2012) Anais do $18^{\circ}$ Workshop de Informática na Escola. Congresso Brasileiro de Informática na Educação. Rio de Janeiro: SBC. Available at< http://www.br-ie.org/pub/index.php/wie/index >, 
access on 10 May 2013.

[30] Schildt, H. (1996) C Completo e Total. São Paulo: Pearson do Brasil.

[31] Schneider, H. N., Nunes, M. A. S. N., Silveira, I. F., Bittencourt, I. I., Machado and G. J. C. (orgs) (2011) Anais do $22^{\circ}$ Simpósio Brasileiro de Informática na Educação e do 17o. Workshop de Informática na Escola. Congresso Brasileiro de Informática na Educação. Rio de Janeiro: SBC. Available at <http://www.br-ie.org/sbiewie2011/\#>, access on 10 May 2013.

[32] Shlaer-Mellor Lee, M. M. (1996) Object Oriented Development: A Manager's Practical Guide. Disponível em http://www.projtech.com.

[33] Siqueira, S. W. M. and Costa, R. M. E. M. (orgs) (2012) Anais do 23 Simpósio Brasileiro de Informática na Educação. Congresso Brasileiro de Informática na Educação. Rio de Janeiro: SBC. Available at <http://www.brie.org/pub/index.php/sbie/index $>$ access on 10 May 2013.

[34] Sommerville, I. (2007) Engenharia de Software. $8^{\mathrm{a}}$ ed. São Paulo: Pearson Addison-Wesley.

[35] Summerfield, M. (2007) Rapid GUI Programming with Python and Qt: The Definitive Guide to PyQt Programming. New Jersey: PrenticeHall.

[36] Tajra, S. F. (2008) Informática na Educação. São Paulo: Érica.

[37] Thiollent, Michel J. M (1985). Metodologia da pesquisa-ação na instituição educativa. São Paulo: Cortez Editora.

[38] Thiollent, Michel J. M (1998) Metodologia da pesquisa-ação. São Paulo: Cortez.

[39] Tkach, D., Puttick, R. (1994) Object Technology in Application Development. The Benjamin/Cummings Publishing Company.

[40] Trucano, M. (2005) Knowledge Maps: ICTs in education. Washington, DC: infoDev/World Bank. Available at <http://www.infodev.org/en/Publication.154.htm 1>, access on 12 June 2012. 